\title{
HACIA UNA PSICOLOGÍA DEL VIVIR COTIDIANO
}

\author{
Charlotte Højholt y Ernst Schraube \\ Roskilde University \\ Dinamarca
}

Traducido de:

\section{INTRODUCTION: TOWARD A PSYCHOLOGY OF EVERYDAY LIVING Charlotte Højholt y ERNSt Schraube}

\author{
Routledge \\ 2016 \\ pp. 1-14 \\ Traducción realizada por: \\ Gilberto Pérez-Campos
}

RESUMEN

Desde su formación como disciplina independiente, la psicología ha estado preocupada con la auto-reflexión humana, pero no ha sido de mucha importancia la pregunta de cómo conducir la propia vida. Con el telón de fondo del trabajo de Max Weber y los sociólogos orientados al sujeto, los psicólogos críticos han incorporado en años recientes el concepto de conducción de la vida cotidiana en la teoría y práctica psicológica. El concepto trata de superar el individualismo abstracto de la psicología (y su acompañante falta de mundo) donde los sujetos están encerrados en funciones psicológicas especiales aisladas, y trata de contribuir a una epistemología psicológica que capta la riqueza, complejidad y conectividad de los fenómenos psicológicos tal y como se despliegan en la vida cotidiana. Este artículo presenta las dimensiones centrales del concepto, lo sitúa en la historia de la psicología y describe un cuerpo de trabajo que analiza con él la práctica contemporánea del vivir cotidiano.

From its formation as an independent discipline, psychology has been concerned with human self-reflection, but the question of how to conduct one's life has not been of much significance. On the background of the work of Max Weber and subject-orientated sociologists, critical psychologists in recent years incorporated the concept conduct of everyday life into psychological theory and practice. The concept tries to overcome the abstract individualism of psychology (and the accompanying wordlessness) where subjects are enclosed in isolated psychological special functions, and tries to contribute to a psychological epistemology, which grasps the richness, complexity and connectedness of psychological phenomena as they unfold in everyday practice. This article presents central dimensions of the concept, situates it in the history of psychology and outlines a body of work analysing with the concept the contemporary practice of everyday living.

Bitácora del Artículo:

| Recibido: 16 de Enero de 2019 | Aceptado: 27 de Marzo de 2019 | Publicado en línea: Julio - Diciembre de 2019 | 


\title{
Autoría y Derechos de Propiedad Intelectual
}

\section{HACIA UNA PSICOLOGÍA DEL VIVIR COTIDIANO}

\author{
Charlotte Højholt y Ernst Schraube \\ Roskilde University \\ Dinamarca
}

Traducido de:

\section{INTRODUCTION: TOWARD A PSYCHOLOGY OF EVERYDAY LIVING Charlotte Højholt y ERNSt SChraube}

\section{Routledge}

2016

pp. $1-14$

Traducción realizada por:

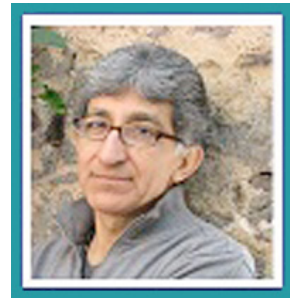

Gilberto Pérez-Campos

UNAM - FES Iztacala

Correo: gpc.fesi@gmail.com

Profesor asociado en la FES Iztacala, UNAM. Es parte de un grupo de investigación que estudia la vida de las personas, principalmente en los contextos familiar y escolar, desde una pespectiva sociocultural.

Ver más...

\section{DATOS DE FILIACIÓN DEL TRADUCTOR}

Profesor titular FES Iztacala, UNAM

\section{(c)

Copyright: (c) 2019 Pérez Campos, G.

Este es un artículo de acceso abierto distribuido bajo los términos de la licencia Creative Commons Reconocimiento-NoComercial 4.0 Internacional, por lo que su contenido gráfico y escrito se puede compartir, copiar y redistribuir total o parcialmente sin necesidad de permiso expreso de su autor con la única condición de que no se puede usar con fines directamente comerciales y los términos legales de cualquier trabajo derivado deben ser los mismos que se expresan en la presente declaración. La única condición es que se cite la fuente con referencia a la Revista Digital Internacional de Psicología y Ciencia Social y a su autor. 


\section{INTRODUCCIÓN ${ }^{1}$}

\section{$\int$}

a cuestión de cómo los seres humanos conducen su vida cotidiana no ha recibido mucha atención Sin embargo, se trata de un asunto crucial, conceptualmente, para explorar y entender la manera como los sujetos individuales organizan, integran y hacen sentido de la multiplicidad de relaciones sociales y demandas contradictorias en y a través de los diferentes contextos en los que están involucrados en su vida diaria.

Este libro es sobre el estudio psicológico del conducir $^{2}$ la vida cotidiana en la sociedad contemporánea. Presenta un cuerpo de trabajo crítico e interdisciplinario, que saca la teoría psicológica y la práctica de la investigación del laboratorio hacia el mundo real. Con su enfoque en la cuestión de cómo los seres humanos, como sujetos sensuales activos, viven sus vidas cotidianas, explora el conducir la vida como base para entender los dilemas y contradicciones con los que nos confrontamos en nuestras vidas diarias.

Desde los primeros días de su formación como una disciplina independiente, la psicología ha estado interesada en la experiencia, la actividad y la auto-reflexión humanas, pero la cuestión de cómo conducir la propia vida no ha recibido mucha atención. La percatación cada vez mayor de esta discrepancia, durante los últimos años, ha dirigido las discusiones dentro de la psicología hacia la práctica del vivir cotidiano y el concepto de conducir la vida cotidiana se está incorporando en la teoría psicológica y la práctica de investigación. La importancia para la psicología de la conducción de la vida cotidiana, radica en su relevancia conceptual para explorar y entender las actividades diarias de los sujetos individuales para organizar, integrar y hacer sentido de la multiplicidad de relaciones sociales y demandas contradictorias en y a través de los diferentes contextos en los que están involucrados en su vida diaria. El concepto

1 Introduction: Toward a psychology of everyday living. En E. Schraube y Ch. Høhjolt (eds.), Psychology and the conduct of everyday life (pp. 1-14). Londres y Nueva York: Routledge, 2016. Traducción realizada por Gilberto Pérez Campos, con autorización de los autores, quienes son titulares de los derechos en cualquier lengua no-inglesa.

2 He traducido el término conduct como "conducir" o como "conducción" (nunca como conducta, para que no haya confusión con behavior); en ambos casos, el énfasis de los términos está en la acción, aunque el último también incluye el efecto de la acción (N. del T.). toma en cuenta cómo las personas producen y reproducen colaborativamente su vida a través de las actividades, hábitos, rutinas y arreglos personales cotidianos de las cosas y las relaciones sociales. El concepto dirige la atención hacia las condiciones sociales en y con las que las personas actúan, participan y viven su vida cotidiana, e incluye la cuestión de cómo las personas están sujetadas a las disposiciones socio-materiales del poder, el conocimiento y el discurso. Además, facilita una exploración de nuevas maneras de vida cotidiana emergentes y cómo éstas contribuyen a rehacer el mundo social. Por lo tanto, trabajar con el "conducir la vida cotidiana" y refinar este concepto puede apoyar una comprensión de los fenómenos psicológicos conforme se desarrollan poco a poco en la realidad del vivir cotidiano, y promover una renovación fundamental de la teoría, la metodología y la práctica psicológica.

En lo que sigue, primero indicaremos cómo el estudio del conducir la vida cotidiana se erige sobre el estudio de la vida cotidiana, así como el modo en que un giro hacia una psicología del vivir cotidiano se relaciona con la auto-comprensión científica de la psicología convencional y qué clase de desafíos teóricos hace surgir. Luego describimos con más detalle la práctica del vivir cotidiano, incluyendo su carácter conflictual y coordinación colectiva, y finalmente presentamos un bosquejo de los diferentes capítulos del libro.

\section{Formación y desafíos de una psicología del vivir cotidiano}

Desde mediados del siglo XX, cuando los estudiosos Ilegaron a reconocer la significación central de la vida cotidiana para comprender los asuntos humanos, el estudio de la vida cotidiana se ha convertido en una preocupación principal dentro de las ciencias sociales y humanas. En vez de consistir la vida cotidiana meramente de actividades triviales y banales del día-a-día, los estudiosos se han dado cuenta que constituye el núcleo de la vida humana y el espacio en que son producidas y reproducidas nuestras relaciones sociales. La vida cotidiana comprende todas las actividades humanas situadas en y a través de una multiplicidad de espacios y contextos (hogar, trabajo, instituciones educativas, sitios de compra, terrenos recreativos, espacios digitales, etc.). Como explica Henri Lefebvre en su trabajo seminal Crítica de la vida cotidiana (1947/1991):

La vida cotidiana está profundamente relacionada con todas las actividades, y las abarca con todas sus diferencias y sus conflictos; es su lugar de encuentro, su vínculo y su terreno común. Y es en la vida cotidiana que la suma total de relaciones que hacen de 
lo humano un todo - y a cada ser humano - toman su figura y su forma (p. 97).

Durante el siglo veinte, partiendo de las realidad de las vidas de las personas, el estudio de la vida cotidiana emergió como un tema de investigación definido a través de una variedad de disciplinas (p.ej., historia, sociología, filosofía, antropología), así como de programas interdisciplinarios (p.ej., estudios culturales, estudios de los medios), desarrollando un vocabulario detallado para explorar el mundo cotidiano en sus complejas y problemáticas relaciones socio-históricas (De Certeau, 1984; Ferguson, 2009; Gardiner, 2000; Goffman, 1959; Highmore, 2010; Pink, 2012; Sheringham, 2006; Smith, 1987, y muchos otros).

El estudio del conducir la vida cotidiana se construye sobre este cuerpo sustancial de trabajo y lo expande con un nuevo enfoque. Basándose en un "giro hacia el sujeto" y en complejas conceptualizaciones de la subjetividad y el ser humano (p.ej., Blackmann et al., 2008; Butler, 1989; Holzkamp, 2013; Walkerdine, 2002), se concentra aún más en la actividad humana y se centra sistemáticamente en la experiencia vivida, la agencia y los esfuerzos de cómo los sujetos humanos viven su vida cotidiana. Esto conlleva un cambio de foco hacia el estudio del vivir cotidiano desde la perspectiva y punto de vista de los sujetos humanos. Estructurando la investigación a través de las experiencias e involucramiento de los sujetos humanos, se puede tratar más consistentemente la complejidad y la naturaleza problemática de la vida cotidiana. Como resultado de esto, se vuelve posible explorar lo que significa el mundo para la vida humana a través del vivir cotidiano y considerar cómo puede ser transformado para mejor.

¿Por qué le tomó tanto tiempo a la psicología dedicarse al conducir la vida cotidiana como un tópico principal de investigación? Puede hallarse una razón en la epistemología particular y la auto-comprensión científica de la psicología convencional. En la investigación empírica convencional, la producción de conocimiento está situada en un escenario específico. Para investigar los fenómenos psicológicos, el investigador construye una situación artificial - típicamente un experimento en un laboratorio universitario. Esta construcción establece una dicotomía entre esta realidad experimental y la realidad cotidiana. Por lo tanto, mientras el arreglo artificial de la realidad experimental reduce la complejidad del asunto de investigación para ajustarse al escenario de laboratorio controlado y manipulable, esto deja abierta la cuestión de qué tanto el conocimiento de los fenómenos psicológicos producido en esta realidad duplicada puede ser transferi- do y generalizado a la realidad de la vida cotidiana (Holzkamp, 2015, cap. 3 en este volumen).

Dicha práctica de investigación psicológica puede verse como la base para un conocimiento psicológico y una construcción de teoría notablemente abstractos, puesto que nos confrontan con el dilema de conocer de qué son parte y con qué están conectados el fenómeno y los problemas estudiados. Aquí, el interés en la vida cotidiana pone de cabeza el esfuerzo de aislar "factores" y "variables", apuntando en su lugar a la necesidad de explorar cómo los fenómenos y los problemas psicológicos están dotados de contenido e importancia precisamente a partir de los contextos de los que son parte. Por ejemplo, las maneras personales de involucrarse en y experimentar los dilemas están conectadas con prácticas, relaciones, preocupaciones y estructuras sociales particulares. El concepto de conducir la vida cotidiana puede abrir entonces una ruta para superar el individualismo abstracto de la psicología (y su ausencia de mundo [worldlessness] asociada) que encierra a los sujetos en funciones psicológica aisladas, y contribuir a una epistemología psicológica que capta la riqueza, complejidad y conexión de los fenómenos psicológicos así como las interrelaciones entre los sujetos humanos y el mundo. Trabajar con esta preocupación implica situar la investigación empírica y la producción de conocimiento psicológico en la realidad cotidiana en la que se desarrollan de verdad los fenómenos psicológicos.

Los psicólogos se volvieron cada vez más conscientes de estos dilemas epistemológicos en las prácticas de investigación psicológica durante los años 1970, en particular a través de la discusión de la crisis de la psicología social (Parker, 1989). Las raíces históricas de esta discusión se pueden rastrear en el trabajo temprano de Wilhelm Dilthey, Lev Vygotski, Wilhelm Stern, Kurt Lewin y muchos otros. Pero la discusión de los años 1970 indujo nuevos comienzos y surgió una variedad de aproximaciones psicológicas alternativas, que iban de la psicología feminista, la fenomenología, la psicología discursiva y el construccionismo social hasta la teoría cultural histórica de la actividad y las psicologías críticas, las cuales sacaron la investigación psicológica de la realidad duplicada del escenario de investigación clásico hacia la realidad de la vida cotidiana (p.ej., Argyle, 1992; Brinkmenn, 2012; Burkitt, 2004; Gergen, 2009; Hodgetts et al., 2010; Scheibe, 2000; Shotter, 1993; Stephenson y Papadopoulos, 2006). Dentro de este amplio movimiento e inspirados por sociólogos orientados hacia el sujeto (Jurczyk y Rerrich, 1993; Voß, 1991; Jurczyk et al., 2015, cap. 2 en este volumen), un grupo de psicólogos críticos puso cada vez más atención a la importancia del 
conducir la vida cotidiana para la psicología, y empezó a explorar sistemáticamente su formación. Como explica Klaus Holzkamp: "El conducir la vida cotidiana es... la forma elemental de existencia humana" (2013, p. 314), y sugiere definir el objeto de estudio constitutivo de la psicología no como el individuo, ni al sujeto individual en las relaciones sociales, sino "el sujeto dentro del contexto de conducir su vida cotidiana" (Ibíd., p. 314).

El concepto de conducción de la vida cotidiana ofrece una aproximación para superar una variedad de desafíos internos fundamentales de la psicología, discutidos desde diferentes ángulos y antecedentes teóricos en los capítulos de este libro. En esta discusión, algunos de los desafíos centrales que surgen conciernen al individualismo crónico del conocimiento psicológico, así como a la cuestión de su relevancia social. ${ }^{3}$

Aunque Wilhelm Wundt, uno de los padres fundadores de la psicología académica, enfatizó que la psicología trata con las experiencias y actividades de los sujetos humanos en relación con otros y con el mundo (1897, p. 3), los modelos explicativos convencionales en la historia de la psicología han favorecido consistentemente un individualismo radical. Como señala James Wertsch: dentro de la psicología contemporánea "la investigación está basada a menudo en la suposición de que es posible, incluso deseable, estudiar al individuo... en aislamiento" (1991, p. 2); a pesar de la "crítica" a lo largo de las décadas, la "orientación individualista es característica de la disciplina en general" (Ibíd., p. 3). Además, explica:

Podemos responder preguntas detalladas sobre la actividad neuronal o los reflejos neonatales, pero tenemos muy poco que decir sobre los que significa ser humano en el mundo moderno ... La psicología se ha vuelto cada vez menos capaz de ofrecer penetración en los principales problemas sociales del día. (Ibíd., p. 1n)

Desarrollar un concepto de la conducción de la vida cotidiana aborda este desafío abriendo una aproximación específica para entender la relación entre el individuo y la sociedad. Dado que la conducción de la vida se refiere a la actividad integrativa y constructiva del sujeto, va más allá de una perspectiva determinista que meramente conceptualiza al individuo como un conjunto de variables dependientes de las estructuras sociales. Por un lado, la cuestión de la conducción de

\footnotetext{
3 Los autores usan a lo largo del artículo los términos "societal" y "social", para el primero de los cuales no tenemos equivalente en castellano (aunque ya es común el uso del neologismo). Revisando las definiciones de ambos en diccionarios de inglés, sus acepciones se traslapan en buena medida. En su uso, el primero intenta resaltar algo que es propio de la sociedad en su conjunto, para distinguirlo de la interacción entre personas. En castellano, ambos sentidos son abarcados por el término social, que usaré para traducirlos (N. del T.).
}

la vida no puede estar limitada a cómo las personas se ajustan a ciertas condiciones dadas, puesto que vivir la propia vida implica arreglar las condiciones, junto con otros, revisar planes y perseguir intereses percibidos. Por otro lado, este concepto va más allá de la perspectiva voluntarista de individuos autónomos y reconoce que la vida individual no está sólo determinada como a cada persona le gustaría, sino que es co-creada a través del tejido del mundo social. La conducción de la vida cotidiana representa una categoría mediadora entre los sujetos individuales y las estructuras sociales, que articula en particular las experiencias de los sujetos y el ámbito de acción conforme luchan con estas estructuras a través de acciones colectivas y estructurantes. Como señala Ole Dreier, un cambio hacia la conducción de la vida cotidiana nos permite mover nuestros "análisis más cerca de lo que significa y toma ser un sujeto que vive en una sociedad" (2015, cap. 1 en este volumen; ver también Dreier, 2008).

El interés en el estudio de la conducción de la vida cotidiana está relacionado con las transformaciones actuales en la sociedad. La globalización, las crisis financiera y ambiental, la deuda, la migración, la guerra y los conflictos a gran escala influencian y cambian los patrones de vida social e individual en todo el mundo (p.ej., Caffentzis, 2015, cap. 9 en este volumen; Federici, 2015, cap. 10 en este volumen). Como resultado de estos cambios, la teoría, la investigación y la práctica psicológicas confrontan nuevos problemas y desafíos complejos. Los psicólogos ahora están lidiando con personas cuyas realidades sociales y formas de vida están sufriendo cambios hacia un individualismo creciente, así como mayor heterogeneidad y complejidad social y cultural. Esto prepara el terreno para que surjan nuevos problemas complejos a través de los avances de las tecnologías, la digitalización, nuevas formas de trabajo y modos de producción, desempleo y pobreza, movilidad individual en aumento, educación para toda la vida y una disposición para el cambio, etc. En este contexto, hay una necesidad cada vez mayor de la que la psicología salga del laboratorio para investigar y entender cómo las personas confrontan y experimentan cambios locales en relación con los sistemas sociales, las instituciones, las tecnologías y las prácticas de la vida diaria en el curso de su vida cotidiana. La teoría y la investigación psicológica tienen, a su vez, que relacionar su comprensión de las actividades y experiencias sensuales con las prácticas y estructuras sociales en que viven y experimentan sus problemas las personas. En este punto, las teorías desarrolladas sobre cómo las personas conducen sus vidas necesitan ser sensibles a los movimientos, trayectorias y 
cambios complejos, así como a las condiciones de vida históricas, culturales, locales y globales. Además, esto requiere reflexionar sobre las nuevas metodologías y prácticas de investigación que facilitan la investigación empírica de las realidades y problemas cotidianos en las vidas de las personas.

\section{La práctica del vivir cotidiano}

Conceptuar la conducción de la vida cotidiana involucra tres dimensiones esenciales. Las actividades humanas con frecuencia llegan a verse como "cotidianas" cuando son procesos que involucran una repetición diaria tal como, por ejemplo, levantarse en la mañana, hacer el desayuno, discutir quién va a comprar los alimentos para la comida, llevar a los niños a la guardería, ir a trabajar, etc. Esta elemental organización cíclica del vivir cotidiano, que incluye ritmos, rutinas y hábitos, constituye una dimensión fundamental de la conducción de la vida cotidiana. Sin embargo, estos ritmos y rutinas de la vida diaria no son sólo mecanismos y no suceden automáticamente, sino que son logros activos de un sujeto individual, dado que incluyen no sólo organizar y resolver tareas y demandas diarias de carácter práctico, sino también coordinarlas e integrarlas en un arreglo total. Karin Jurczyk, G. Günter Voß y Margit Weihrich han descrito la conducción de la vida como el "arreglo de los arreglos individuales" (2015, cap. 2 en este volumen, p. 46). Los ritmos y las rutinas nos eximen de la necesidad de observar, negociar y justificar constantemente lo que hacemos o no hacemos, y proveen un fundamento básico a nuestras vidas.

Sin embargo, conducir la vida cotidiana involucra más que la repetición ordinaria y la estructura cíclica del vivir cotidiano. La práctica del vivir cotidiano requiere no sólo una dimensión reproductiva, sino también una productiva, donde trascendemos las rutinas y los ciclos ordinarios ya sea deliberadamente o a través de la necesidad. La dimensión productiva nos permite organizar y asumir las demandas, perturbaciones y desafíos diferentes, contradictorios y a menudo imprevisibles con los que nos enfrentamos en los diversos terrenos de nuestra vida diaria. Por lo tanto, la conducción de la vida cotidiana también involucra siempre una dimensión de trascendencia y experiencia y actividad extra-ordinaria, situaciones en el vivir cotidiano donde entramos en un territorio desconocido, nos enfrentamos a desafíos y luchas serias, experimentamos momentos de felicidad, gozo o comprensión excepcional (o lo opuesto) en los que podemos incluso tener la impresión de que estos momentos extra-ordinarios son precisamente donde ocurre "realmente" la vida.
Ambos aspectos, lo ordinario cíclico y lo trascendente extra-ordinario, del vivir cotidiano están integrados en una tercera dimensión central de la conducción de la vida cotidiana. Vivir la propia vida incluye lo que podríamos llamar la sensibilidad integrativa y manera de hacer sentido específica del sujeto, sin la cual la conducción de la vida no sería posible. Por lo tanto, conducir la vida no se refiere sólo a la organización día-a-día y a la integración de las diversas demandas, sino que está también conectada a la concepción del sujeto de su historia así como a su futuro. Está relacionada (y podemos no percatarnos siempre de ello) con una imaginación más amplia de cómo vemos el mundo y qué queremos con nuestra vida, y a partir de esta imaginación más amplia de nuestra vida y la anticipación de las posibilidades de acción, las actividades y arreglos cotidianos concretos ordinarios y extra-ordinarios se vuelven significativos y alcanzables. La experiencia personal de cohesión en la vida también está relacionada con los conflictos sociales, por ejemplo entre demandas contradictorias en la vida, y cómo tales conflictos se desarrollan, se desanudan o llegan a un impasse. En lo que sigue, discutimos con más detalle cómo el hacer sentido personal está entrelazado con la conflictividad social de la conducción de la vida cotidiana.

Todas las diferentes aproximaciones que se presentan en este libro discuten la conducción de la vida cotidiana como un proceso fundamentalmente colectivo. El concepto de conducir la vida se aplica y desarrolla analizando cómo los sujetos conducen sus vidas en colaboración con otros sujetos y en relación con diferentes asuntos en sus vidas. Tal conceptualización busca captar la dimensión inherentemente social de nuestras vidas como seres humanos y, de esta manera, considerar al sujeto en plural. Este enfoque apunta a la coordinación y los conflictos sociales como los problemas centrales de la conducción personal de la vida (Axel, 2011; Chimirri, 2014). Desplazar la noción de conducción de la vida de las vidas de personas singulares hacia los procesos de organizar vidas diferentes pero entretejidas, cambia nuestra comprensión de las rutinas en la vida cotidiana. DesarroIlar rutinas no es sólo un proyecto individual. Nuestras rutinas son creadas, mantenidas y negociadas en el contexto de la coordinación con la conducción de la vida de otros, lo cual puede también conllevar conflictos o luchas con ellos. Aquellos involucrados en vidas cotidianas mutuas - sea como miembros de la familia, colegas, vecinos o amigos - cooperan o disienten acerca de cómo establecer preferencias, hacer planes, priorizar y llevar a cabo rutinas cotidianas simples, así como en las alteraciones, reconstrucciones y desvíos más abarcadores. 
En los países occidentales de hoy, las personas viven sus vidas cotidianas en una pluralidad de contextos. En estos diferentes contextos, las vidas de los participantes están conectadas entre sí de maneras fundamentalmente diferentes, y sus actividades están estructuradas en relación con diferentes propósitos y condiciones. En estas prácticas interconectadas, los problemas en un lugar (p.ej., en la familia) están relacionados a menudo con conflictos entre diversos contextos (p.ej., escuela y hogar, o diferentes lugares de trabajo) donde los involucrados toman parte. Así, en la trayectoria a través de los contextos cotidianos, tienen que tomarse en cuenta muy diversos asuntos y preocupaciones y relacionarse entre sí. Por lo tanto, cuando las personas conducen sus vidas cotidianas están tomando parte en reproducir, negociar y cambiar las estructuras sociales, y organizar las vidas involucra explorar posibilidades estructurales, conexiones y restricciones en la vida cotidiana. De esta manera podemos investigar las estructuras sociales a través de las maneras personales en que los sujetos se relacionan y asignan significados a éstas en el vivir cotidiano - tal y como se expresa en su participación y experiencia personal así como en sus dilemas y conflictos.

Analizar tales aspectos del vivir cotidiano se construye a partir de las comprensiones teóricas de investigación que resalta la naturaleza cultural e histórica de los procesos sociales (p.ej., Chaiklin, Hedegaard y Jensen, 1999; Holland y Lave, 2001; Juul Jensen, 1999; Lave, 2008, 2011). Tal aproximación señala la naturaleza del vivir cotidiano como fundamentalmente conflictiva, en vez de considerar el conflicto como una experiencia intermitente vinculado con perturbaciones o errores. Puesto que el conflicto se considera como una parte inherente de las prácticas sociales, esta conflictividad genera/ puede verse como un trasfondo analítico para entender los dilemas específicos de la conducción de la vida cotidiana.

Enfocarse en la conducción de la vida cotidiana abre perspectivas y preocupaciones personales y sociales, así como políticas. La individualización cada vez mayor de instituciones clave (como la familia, la educación y el trabajo) y de las maneras de organizar la vida social impone nuevas demandas a nuestra conducción de la vida cotidiana. Contra este trasfondo, un interés académico en el concepto puede tender entonces a gravitar hacia los discursos individualistas de la vida humana, en los que el conocimiento de la conducción de la vida parece ofrecer el potencial de monitorear, disciplinar y regular las vidas de los otros. Sin embargo, es precisamente el conocimiento de cómo las personas experimentan estas ideologías y cambios sociales en la conducción de sus vidas cotidianas, y cómo trabajan para transformarlas, el que podría revelar también las fuerzas de la individualización y del gobernar a los otros.

Un punto de referencia en la vida común que los sujetos viven en un mundo compartido ilustra cómo los intereses y las perspectivas son no sólo diferentes, sino también están conectados y relacionados a través de asuntos, preocupaciones y problemas conjuntos pero multifacéticos. Los conflictos de los participantes son una expresión de ese compromiso compartido y ofrecen una oportunidad para ampliar nuestra comprensión sobre los problemas comunes (Busch-Jensen, 2015). De esta manera, un concepto de la conducción de la vida cotidiana potencialmente puede derivar conocimiento de las diferentes experiencias de las personas de los dilemas en su vida cotidiana - no como conocimiento sobre "otras personas" sino conocimiento sobre los problemas sociales vistos desde estas perspectivas personales (elaborado en Kousholt, 2015, cap. 13 en este volumen, p. 245n). Indagando en los significados, razones y conflictos involucrados en el vivir cotidiano, la psicología puede lograr una nueva relevancia en explorar, entender y transformar los problemas sociales. Necesitamos conectar los diferentes aspectos de preocupaciones multifacéticas en vez de reducir su complejidad a dicotomías entre "intereses sociales" e "intereses individuales". De esta manera, el concepto de vida cotidiana puede ofrecer opciones para la solidaridad y para una metodología de investigación democrática donde los problemas comunes se exploren a través de la manera en que son experimentados en el vivir cotidiano y desde diferentes ubicaciones y posiciones. Aquí, las contribuciones en este libro pretender avanzar más esta aproximación involucrándose en diálogos críticos sobre cómo las personas buscan activamente crear y conducir sus vidas en y a través de las complejas realidades de la vida cotidiana de hoy en día, y también considerar las discusiones y conceptualizaciones teóricas relacionadas.

Los capítulos siguientes ilustran, de varias maneras, un avance hacia una comprensión situada, social y contextual de los procesos psicológicos. Varios capítulos, por ejemplo, subrayan la implicación de que son necesarias prácticas metodológicas en las que los investigadores participen en las prácticas cotidianas y cooperen con quienes están fuera de la academia como "co-investigadores" en una exploración compartida de los problemas sociales (en vez de posicionar al "otro" como objeto de la investigación). Tales metodologías conceptualizan maneras flexibles de "entrar en la vida cotidiana" a través de diversos modos de participación, enriqueciendo a los investigadores con una comprensión situada de perspectivas mundanas y a menudo inadvertidas o inclu- 
so desatendidas. En palabras de Darrin Hodgetts, Mohi Rua, Pita King y Tiniwai Te Whetu:

El estilo de investigación en el que nos comprometimos en nuestro proyecto funciona de una manera más flexible, en la que tratamos más duro de acomodarnos en los eventos cotidianos... Una metodología basada en casos caracterizada por relaciones más cercanas y más comprometidas entre investigadores y participantes de la que es típicamente obvia en la psicología. (2015, cap. 6 en este volumen, pp. 141-142).

En el mismo sentido, este volumen intenta corroborar una variedad de aproximaciones situadas críticamente hacia las experiencias y acciones de conducción de la vida en el mundo social y tecnológico de hoy, y debatir cómo el estudio de los sujetos en el contexto de la conducción de su vida cotidiana puede contribuir a la renovación de la teoría, la metodología y la práctica psicológica. ¿Cómo podemos desarrollar el conocimiento sobre y la investigación de los esfuerzos activos de los sujetos para involucrarse en las actividades, tareas y participaciones diarias a través del tiempo y el espacio? ¿Qué clases de nuevas formas de hacer y pensar en la vida cotidiana están surgiendo y cómo contribuyen a rehacer el mundo social? ¿Cómo podemos reconceptualizar la subjetividad, la agencia y las posibilidades de cambio social y político? Los trece capítulos en este volumen fueron escritos por académicos distinguidos que trabajan en la psicología y otras ciencias sociales y humanas, quienes están comprometidos en desarrollar este campo de investigación $y$, a través de su trabajo teórico, metodológico y empírico, contribuir al estudio de la conducción de la vida en y a través de diferentes terrenos de la vida cotidiana.

\section{Exploraciones del vivir cotidiano}

En el primer capítulo de este volumen Ole Dreier resalta el poder del concepto de conducción de la vida cotidiana para reformar la mirada psicológica. Se enfoca especialmente en las implicaciones del concepto para la psicología crítica y explica cómo puede permitir un cambio en la aproximación de la psicología crítica a partir de estudiar a los sujetos en su situación de vida inmediata vis-à-vis una estructura social total para estudiar cómo conducen sus vidas en estructuras de práctica social cotidiana. Tal cambio, argumenta, cambiará cómo entendemos el funcionamiento psicológico de las personas, sus experiencias, preocupaciones, posturas y auto-comprensión. También cambia cómo entendemos la importancia de la estructura social total para los sujetos considerándola desde el punto de vista y perspectiva de personas que conducen sus vidas en estructuras de práctica social.
En el capítulo 2, Karin Jurczyk, G. Günter Voß y Margit Weihrich introducen una aproximación sociológica para el estudio de la conducción de la vida cotidiana. Describen el desarrollo de su cuerpo de investigación a lo largo de los años, conceptuando la conducción de la vida como el esfuerzo discreto hecho por el individuo para satisfacer las diferentes - y a veces conflictivas -demandas que surgen en las diversas esferas de la vida cotidiana, y para organizarlas en un todo vivible. Por lo tanto, la sociedad puede entenderse a través de las vidas cotidianas de los individuos que realizan estas acciones. En la presentación de su extensa investigación empírica sobre las formas, métodos, recursos y esfuerzos del conducir la vida cotidiana bajo una variedad de condiciones de vida, ilustran cómo conducir la propia vida en el proceso de modernización se está convirtiendo en un desafío siempre en aumento.

El capítulo 3 presenta un manuscrito no terminado de Klaus Holzkamp sobre la conducción de la vida cotidiana sobre el que estaba trabajando antes de su muerte en 1995 (el texto se publicó en alemán en la revista Das Argument, existe un segundo texto manuscrito inconcluso por Holzkamp sobre la conducción de la vida publicado en inglés en 2013). Durante su investigación sobre el aprendizaje Holzkamp se percató de la importancia de la vida cotidiana para la psicología. Se dio cuenta de que en toda la historia de la psicología la conducción de la vida cotidiana no había sido analizada o conceptualizada de ninguna manera sistemática y [conceptualizado] como un problema teórico por derecho propio. Construyendo sobre el trabajo del grupo de sociólogos orientados al sujeto alrededor de Jurczyk, Voß y Weihrich, empezó a [profundizar] en una exploración de la conducción de la vida cotidiana. Este capítulo presenta algunas de las primeras comprensiones sobre esta ruta, incluyendo una discusión de la aproximación sociológica, una análisis de por qué el concepto no ha sido investigado hasta ahora en la psicología, así como una reflexión de cómo estudiar la conducción de la vida cotidiana desde el punto de vista del sujeto.

En el capítulo 4 el antropólogo Tim Ingold se enfoca en la importancia de una práctica específica del vivir cotidiano: caminar y la práctica del exponerse como un modelo alternativo de educación. Argumenta en favor de una práctica educativa que más que infiltrar el conocimiento en las mentes de los novicios, los conduce hacia el mundo real. Ingold compara estas alternativas con la diferencia entre el maze y el labyrinth. El primero, que presenta una serie de elecciones pero predetermina los movimientos implicados en cada uno, pone todo el énfasis en las intenciones del viajero. En contraste, en el labyrinth no

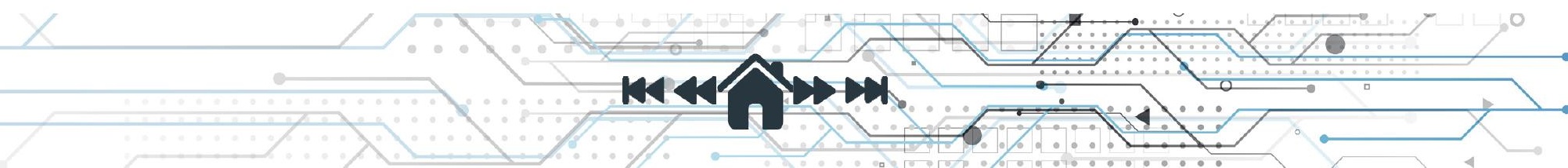


es cuestión de elección, sino que mantenerse en el camino demanda de atención continua. La educación a lo largo de las líneas del labyrinth no provee a los novicios de puntos de vista o posiciones, sino que continuamente los jala desde cualquier posición que puedan adoptar. Es una práctica del exponerse. La atención requerida por tal práctica es una que respeta las cosas y que está presente ante su aparición. "Aparecer cosas" es equivalente a su imaginación, en el plano de la vida inmanente. La vida humana está tensionada entre la imaginación y la percepción, y la educación (en el sentido original del griego scholè) Ilena la brecha entre ellas. El autor concluye que tal "pedagogía pobre" provista por un modo de educación que no tiene contenido que transmitir, ni métodos para hacerlo, ofrece no obstante una comprensión del camino hacia la verdad.

En el capítulo 5, Thomas Teo discute la aproximación de Holzkamp a la conducción de la vida cotidiana en el contexto de la psicología crítica como un programa internacional de investigación progresivo que puede asimilar y acomodar las tradiciones críticas desde dentro y fuera de Occidente. Argumenta que Holzkamp ofrece una solución de primer orden a la relación entre la sociedad y el individuo y una solución parcial al problema de cómo la psicología crítica debería conceptuar la mediación entre la estructura social y la conducción de la vida cotidiana. apoyándose en el trabajo de Pierre Bourdieu, Judith Butler y Peggy Mclntosh, sugiere que el estudio de la conducción de la vida necesita incluir conceptos tales como habitus, performatividad y privilegio, que están fundamentados en teorías críticas de la corporización y no en una filosofía de la conciencia.

El vivir cotidiano se conceptúa a menudo en términos de lo mundano u ordinario. Sin embargo, para un número cada vez mayor de personas la disrupción y lo extra-ordinario se han convertido en normativos. En el capítulo 6, Darrin Hodgetts, Mohi Rua, Pita King y Tiniwai Te Whetu exploran cómo lo ordinario re-emerge dentro de las vidas extra-ordinarias de un grupo de hombres maorí de edad avanzada sin casa cuando pasaban los martes y jueves en un jardín marae (un lugar de reunión tradicional maorí para la conducción de la vida cotidiana). El capítulo ilustra y reflexiona sobre cómo llevar a cabo investigación empírica sobre el vivir cotidiano, y presenta nuevas perspectivas de cómo la jardinería ofrece a los hombres maorí una oportunidad de reafirmar relaciones, herencia e identidades pautadas culturalmente.

Basada en investigación empírica de la conducción de la vida de los niños, Charlotte Høhjolt se enfoca en el capítulo 7 en los conflictos en la vida cotidiana y su relación con los conflictos sociales, políticos y estructu- rales. Con el concepto de desigualdad situada, Ilama la atención hacia la distribución social de las posibilidades para tomar parte en, e influenciar, la coordinación social de los contextos cotidianos. Discutiendo ejemplos de la conducción de la vida cotidiana de niños entre arreglos institucionales, donde diversas partes tienen diferentes clases de responsabilidades y perspectivas en conflicto sobre los niños, argumenta que los conflictos sociales (y políticos) sobre los niños conducen a problemas personales para los niños. De esta manera, los dilemas concretos de los niños nos pueden enseñar algo sobre los conflictos sociales en y sobre la escuela. Finalmente, ella discute los desafíos de cómo conceptuar los significados de los antecedentes sociales de los niños y cómo conceptuar su agencia personal.

El punto de partida de la exploración del vivir cotidiano por Ute Osterkamp en el capítulo 8, es la famosa máxima de Adorno "no hay vida recta en la equivoca$\mathrm{da}^{\prime \prime}{ }^{4}$ y ella argumenta que reconocer este dilema es el primer paso para salir de él. Osterkamp indica que la expresión de Adorno a menudo es rechazada con el argumento de que niega cualquier posibilidad de cambio y la responsabilidad de las personas en él. Tales objeciones parecen provenir de una noción restringida de la agencia y la responsabilidad humanas, reduciéndolas a los mejores ajustes posibles a las condiciones y normas dadas. En contraste, ella discute cómo, desde la perspectiva de una psicología conceptualizada desde el punto de vista de un sujeto generalizado, se vuelven visibles tanto la "inhumanidad" de tal visión limitada de la agencia y la subjetividad como la necesidad subjetiva de superar las condiciones inhumanas. Sin embargo, para superar tal concepto restringido de agencia y subjetividad es necesario darse cuenta de las variadas formas y maneras en que inadvertidamente apoyamos en nuestros pensamientos y acciones las condiciones que queremos superar. Esto incluye la necesidad de reconocer y resistir las muchas presiones que nos llevan a ignorar toda la información contradictoria como para mantener la apariencia de ser capaces de vivir nuestras vidas en la forma correcta, en contraste con otros.

En el capítulo 9 el filósofo George Caffentzis examina la vida cotidiana a la sombra del ascenso de la economía de la deuda en los Estados Unidos. Reconociendo

4 Esta máxima de Adorno, en el original alemán "Es gibt kein richtiges Leben im falschen", se ha traducido de diversas maneras que aunque convergentes en su sentido, tienen diferentes matices: "No hay vida recta en la vida falsa"; "Una vida equivocada no se puede vivir de forma correcta"; "La vida errónea no puede ser vivida correctamente"; "No hay vida real en el mal"; "No hay vida justa en medio de la vida falsa". Elegí una traducción más bien literal de la forma como dicha máxima aparece en inglés en el libro: "there in no right life in the wrong one" (N. del T.). 
que los grandes teóricos de la vida cotidiana (de Marx a Lefebvre) no atendieron al impacto de la deuda, en sus investigaciones Caffentzis articula la nueva estructura de la satisfacción de necesidades que la economía de la deuda impone, que es bastante diferente de la estructura de las necesidades en la economía previa dominada por el salario. Además, él analiza cuatro diferentes clases de relaciones deudor-acreedor con respecto a las relaciones de clase, y reflexiona sobre las nuevas formas de alienación que Marx y los teóricos de la vida cotidiana del siglo XX habían negado. El capítulo concluye con un bosquejo histórico del movimiento anti-deuda en los Estados Unidos que despertó siguiendo los pasos de la crisis financiera de 2008 y que puede desafiar potencialmente la conducción contemporánea de la vida cotidiana en la economía de la deuda.

En el capítulo 10, la activista feminista y filósofa política Silvia Federici explora cómo la subsunción del vivir cotidiano a las necesidades del mercado de trabajo afecta nuestras relaciones sociales y subjetividad, y cómo podemos re-apropiarnos nuestras vidas, nuestros cuerpos y superar la crisis reproductiva que estamos encarando. Ella discute estas cuestiones examinando cómo la reestructuración de la economía mundial ha afectado el trabajo reproductivo y las relaciones de género, el papel que ha jugado la tecnología en este proceso, y las iniciativas que las mujeres en particular están tomando, en todo el mundo, para construir formas más cooperativas y equitativas de reproducción. Ella argumenta que el vivir cotidiano actual debe empezar con una lucha contra el asalto histórico, aun en curso, sobre los medios de nuestra reproducción y nuestro ambiente social y ecológico.

En en capítulo 11, Ernst Schraube y Athanasios Marvakis investigan el significado contradictorio de la tecnología digital en el aprendizaje de los estudiantes. La digitalización de la educación superior está transformando radicalmente el aprendizaje y las relaciones de enseñanza, incluyendo el contenido del aprendizaje y la conducción de la vida de los estudiantes. El capítulo analiza la relevancia de la tecnología digital para los aprendices y sus actividades de aprendizaje enfocándose en la cuestión del aprendizaje. ¿Qué es el aprendizaje? ¿Cómo integrar a los aprendices en la teoría del aprendizaje y en las reflexiones sobre la tecnología digital? ¿Por qué, cómo y para qué fin ocurre el aprendizaje, y cuáles son las mejores condiciones posibles para el aprendizaje? Construyendo sobre la teoría de aprendizaje situado y participativo, el capítulo muestra cómo el aprendizaje es no sólo una transferencia o internalización del conocimiento, sino una actividad humana básica para apropiarse y cambiar el mundo, con su raíz en nuestra conducción de la vida cotidiana. Lucha por conceptuar el aprendizaje desde el punto de vista de los aprendices y describe los elementos decisivos y las fases de la actividad de aprendizaje incluyendo el papel crucial del entrelazamiento mutuo y fluido del aprendizaje y la enseñanza. Basado en un concepto refinado de aprendizaje muestra cómo la digitalización de los ambientes de aprendizaje de los estudiantes reconfigura las estructuras de participación y cómo puede catalizar pero también congelar la fluidez del aprendizaje y la enseñanza.

Apoyándose en los pensamientos de Antonio Gramsci sobre cómo los conceptos teóricos se relacionan con el sentido común, Frigga Haug explora en el capítulo 12 la relación entre teoría y comprensión cotidiana como un desafío básico en el estudio de la conducción de la vida. El capítulo introduce el trabajo-de-memoria como una aproximación posible para tratar con este desafío y presenta sus pasos iniciales, fundamentos teóricos y posibilidades para una indagación empírica del vivir cotidiano. Haug subraya cómo el trabajo-de-memoria puede contribuir a empoderar a los sujetos individuales tomando control de su propia conducción de la vida y presenta el proyecto político Perspectiva-Cuatro-en-Uno. Éste es una propuesta para entender que el vivir cotidiano toma lugar en cuatro áreas principales (vida laboral, reproducción, auto-desarrollo y política) y aborda los desafíos de reunir estas cuatro áreas en una conducción de la vida, en vez de pasar toda nuestra vida en sólo una o dos de estas áreas, infelizmente y subordinado. Finalmente, el capítulo ilustra cómo corroborar tal proyecto político en un trabajo-de-memoria anticipatorio y un trabajo con esperanza.

En el capítulo 13, Dorte Kousholt amplía la discusión metodológica de cómo explorar empíricamente el vivir cotidiano enfocándose en las interconexiones de los aspectos subjetivos y estructurales de las personas conduciendo su vida cotidiana en y a través de las prácticas sociales. Kousholt discute las posibilidades para organizar una colaboración de investigación participativa que permita el desarrollo de conocimiento sobre los problemas comunes y las condiciones de vida contradictorias en sus significados para diferentes personas. Basada en ejemplos de sus propia investigación, donde siguió las vidas de niños y las transiciones a través de sus diferentes contextos de vida, ella demuestra cómo investigar las prácticas desde diferentes posiciones y perspectivas y cómo esto proporciona conocimiento sobre la complejidad de los conflictos sociales y las posibilidades de cambio.

\section{Referencias}


Argyle, M. (1992). The social psychology of everyday life. London: Routledge.

Axel, E. (2011). Conflictual cooperation. Nordic Psychology, 20 (4), $56-78$.

Blackmann, L., Cromby, J., Hook, D., Papadopoulos, D. \& Walkerdine, V. (2008). Creating subjectivities. Subjectivity, $22,1-27$.

Brinkmann, S. (2012). Qualitative inquiry in everyday life. London: Sage.

Burkitt, I. (2004). The time and space of everyday life. Cultural Studies , 18 (2/3), $211-227$.

Busch-Jensen, P. (2015). The production of power in organizational practice: Working with conflicts as heuristics. Outlines: Critical Practice Studies, 16.

Butler, J. (1989). Gender trouble: Feminism and the subversion of identity. New York: Routledge.

Chaiklin, S., Hedegaard, M. \& Jensen, U. J. (eds.). (1999). Activity theory and social practice. Aarhus: Aarhus University Press.

Chimirri, N. A. (2014). Investigating media artifacts with children: Conceptualizing a collaborative exploration of the sociomaterial conduct of everyday life. Roskilde: Roskilde University.

De Certeau, M. (1984). The practice of everyday life (S. Rendall, trans.). Berkeley: University of California Press.

Dreier, O. (2008). Psychotherapy in everyday life. Cambridg: Cambridge University Press.

Ferguson, H. (2009). Self-identity and everyday life. London: Routledge.

Gardiner, M. E. (2000). Critiques of everyday life. London: Routledge.

Gergen, K. J. (2009). Relational being: Beyond self and community. New York: Oxford University Press.

Goffman, E. (1959). The presentation of self in everyday life. New York: Anchor.

Highmore, B. (2010). Ordinary lives: Studies in the everyday. London: Routledge.

Hodgetts, D., Drew, N., Sonn, C., Stolte, O., Nikora, L. \& Curtis, C. (2010). Social psychology and everyday life. Basingstoke: Palgrave Macmillian.

Holland, D. \& Lave, J. (2001). History in person: Enduring struggles, contentious practice, intimate identities. Santa $\mathrm{Fe}$, NM: School of American Research Press.

Holzkamp, K. (2013). Psychology: Social self-understanding on the reasons for action in the conduct of everyday life. En E. Schraube \& U. Osterkamp (eds.), Psychology from the standpoint of the subject: Selected writings of Klaus Holzkamp (pp. 233-341) (A. Boreham \& U. Osterkamp, trans.). Basingstoke: Palgrave Macmillan.

Jurczyk, K. \& Rerrich, M. S. (eds.). ( 1993 ). Die Arbeit des Alltags: Beitrage zu einer Soziologie der alltaglichen Lebensführung [The work of everyday life: Contributions to a sociology of the conduct of everyday life]. Freiburg: Lambertus.

Juul Jensen, U. (1999). Categories in activity theory: Marx's philosophy just-in-time. En S. Chaiklin, M. Hedegaard \& U. Juul Jensen (eds.), Activity theory and social practice: Cultural-historical approaches (pp. 79-99). Aarhus: Aarhus University Press.

Lave, J. (2008). Situated learning and changing practice. En A. Amin \& J. Roberts (eds.), Community, economic creativity, and organization (pp. $283-296$ ). Oxford: Oxford University Press .

Lave, J. (2011). Apprenticeship in critical ethnographic practice. Chicago, IL: Chicago University Press.

Lefebvre, H. (1991). Critique of everyday life (J. Moore, trans.). London: Verso. (Original work published 1947).

Parker, I. (1989). The crisis in modern social psychology - and how to end it. London: Routledge.

Pink, S. (2012). Situating everyday life: Practices and places. London: Sage.

Scheibe, K. E. (2000). The drama of everyday life . Cambridge: Harvard University Press.

Sheringham, M. (2006). Everyday life: Theories and practices from surrealism to the present. Oxford: Oxford University Press.

Shotter, J. (1993). Cultural politics of everyday life: Social constructionism, rhetoric and knowing of the third kind. Toronto: University of Toronto Press.

Smith , D. E. (1987). The everyday world as problematic . Boston, MA: Northeastern University Press.

Stephenson, N. \& Papadopoulos, D. (2006). Analysing everyday experience: Social research and political change. London: Palgrave Macmillan.

Voß, G. G. (1991). Lebensführung als Arbeit. Über die Autonomie der Person im Alltag der Gesellschaft [Conduct of life as work: On persons' autonomy in the everyday life of society]. Stuttgart: Enke.

Walkerdine, V. (ed.) (2002). Challenging subjects: Critical psychology for a new millennium. Basingstoke: Palgrave Macmillan.

Wertsch, J. V. (1991). Voices of the mind: A sociocultural approach to mediated action. Cambridge: Harvard University Press.

Wundt, W. (1897). Outlines of psychology (C. H. Judd, trans.). New York: Stechert. 


\section{Historia del Proceso} EDITORIAL 